3

4 Haochun Tang ${ }^{\mathrm{a}, \mathrm{b}}$, Chun-Yi Chen ${ }^{\mathrm{ab},{ }^{*},}$, Takashi Nagoshi ${ }^{\mathrm{c}}$, Tso-Fu Mark Chang ${ }^{\mathrm{a}, \mathrm{b}}$,

\section{Enhancement of mechanical strength in Au films electroplated with supercritical carbon dioxide}

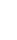
Daisuke Yamane $^{\mathrm{a}, \mathrm{b}}$, Katsuyuki Machida ${ }^{\mathrm{a}, \mathrm{b}, \mathrm{d}}$, Kazuya Masu ${ }^{\mathrm{a}, \mathrm{b}}$, Masato Sone ${ }^{\text {a,b }}$

${ }^{a}$ Institute of Innovative Research, Tokyo Institute of Technology, Yokohama, 226-8503, Japan

14 * Corresponding author: Tel: +81-45-924-5631

E-mail: chen.c.ac@m.titech.ac.jp
${ }^{b}$ CREST, Japan Science and Technology Agency, Yokohama, 226-8503, Japan

${ }^{c}$ National Institute of Advanced Industrial Science and Technology, Tsukuba Ibaraki, 305-8564, Japan

${ }^{d}$ NTT Advanced Technology Corporation, Atsugi Kanagawa, 243-0124, Japan 


\section{Abstract}

The effects of grain refinement and enhancement in the micro-mechanical strength were observed in the $\mathrm{Au}$ film fabricated by electroplating with a supercritical $\mathrm{CO}_{2}$ contained electrolyte (EP-SCE). The ultra-fine grained Au film was achieved when a current density of $5 \mathrm{~mA} / \mathrm{cm}^{2}$ and a pressure of $10 \mathrm{MPa}$ were applied. High carbon content was also observed in the film, which contributed to the grain refinement effect and expected to stabilize the ultra-fine grain structure. Micro-mechanical properties of the Au films were evaluated by micro-compression tests for the applications as the components in micro-electronic devices. Dimensions of the fabricated Au micro-pillar were $10 \times 10 \times 20 \mu \mathrm{m}^{3}$. The $0.2 \%$ yield strength and the compressive flow stress of the Au micro-pillar reached $520 \mathrm{MPa}$ and $800 \mathrm{MPa}$, respectively. The micro-pillar fabricated from the EP-SCE Au film exhibited a much higher mechanical strength than that from the conventional electroplated Au film.

29

Keywords: Gold, electroplating, supercritical carbon dioxide, mechanical property, micro-compression test 


\section{Introduction}

In past few decades, Au materials have attracted much attentions and are widely used in

micro-electronic industries because of their outstanding properties such as high chemical stability, corrosion resistance, electrical conductivity, and density [1,2]. Among the fabrication methods, electroplating (EP) is extensively applied in fabrication of the $\mathrm{Au}$ materials used in micro-electrical-mechanical system (MEMS) devices, i.e. capacitive accelerometers [3]. However, the mechanical strength of $\mathrm{Au}$ is relatively low (i.e., yield strength of bulk Au: 50-200 MPa [4]) when compared with other metallic materials, which has been a concern in practical applications in MEMS especially when used as the movable components. Strengthening by grain refinement is a typical method to enhance the mechanical strength of metallic materials based on the Hall-Petch relationship [5]. As the grain size reaches submicron- or nano-scale, high strength can be obtained when compared to coarse-grained metals with the same chemical composition and phase constitution [6,7].

Grain size control is a fascinating feature in the EP by appropriately adjusting the deposition parameters such as the current density, $\mathrm{pH}$ value, plating bath temperature, etc. [8]. For example, an increase in the current density can lead to a decrease in the grain size, and therefore, enhances the mechanical strength. However, the film composition could be varied because of side-reactions, defects could be formed in the film, and the current efficiency would be low when the current density is too high. Another example would be pulse electroplating (PEP). The grain size can be controlled by adjusting the pulse-on current density and the duty cycle, and therefore, finer grains could be obtained with the optimized PEP conditions $[9,10]$.

On the other hand, an alternative EP method employing a constant current density and a supercritical carbon dioxide $\left(\mathrm{scCO}_{2}\right)$ contained electrolyte was reported to be effective to give the grain refinement effect as demonstrated in electroplating of $\mathrm{Ni}$ and $\mathrm{Cu}[11,12]$. Moreover, the specific properties of $\mathrm{scCO}_{2}$ can promote transfer of materials into confined spaces and 
removal of $\mathrm{H}_{2}$ gas bubbles away from the cathode, which allow application of high current density while keeping a defect- and pinhole-free plating at the same time [13-15]. In addition, co-deposition of carbon was reported in electroplating with an electrolyte containing $\mathrm{scCO}_{2}$ at high pressure (EP-SCE) [16,17]. The co-deposited carbon is effective to cause the grain refinement effect. Also, the carbon would precipitate at the grain boundary to suppress the occurrence of the inverse Hall-Petch relationship [16,18] and reduce the grain boundary mobility [19], which is very effective in stabilizing the ultra-grain structures to maintain the high mechanical strength. With these advantages, mechanical strength of the electroplated Au films in is expected to be enhanced by introducing $\mathrm{scCO}_{2}$ into the $\mathrm{Au}$ EP process to improve the long-term reliability of the Au components used in MEMS devices.

In this work, micro-mechanical properties of the Au film prepared by the EP-SCE were evaluated by micro-compression tests using a non-tapered micro-sized pillar with a square cross-section. The Au micro-pillar was fabricated from the electroplated film by focus ion beam (FIB). The present results showed that the Au film fabricated by the EP-SCE exhibits higher mechanical strength than that of the conventional EP (CONV-EP) film fabricated without using $\mathrm{scCO}_{2}$.

\section{Experimental}

\subsection{Electroplating process}

The Au electrolyte used in this work was a commercially available sulfite-based electrolyte provided by METEX Corporation, Japan, which contained $50 \mathrm{~g} / \mathrm{L}$ of $\mathrm{Na}_{2} \mathrm{SO}_{3}, 50$ of g/L $\left(\mathrm{NH}_{4}\right)_{2} \mathrm{SO}_{3}, 10 \mathrm{~g} / \mathrm{L}$ of $\mathrm{Au}$, and $5 \%$ sodium gluconate with $\mathrm{pH}$ of 8.0 . Cu plates and $\mathrm{Pt}$ plates were used as the cathode and anode, respectively. Two methods were employed for preparation of the Au films: one was the CONV-EP without employing $\mathrm{scCO}_{2}$. The other was the EP-SCE. For the EP-SCE, 20 vol.\% of $\mathrm{CO}_{2}$ with respect to the overall volume of the reaction chamber was used. The pressure was controlled at $10 \mathrm{MPa}$ during the entire EP-SCE 
process. The current density and the plating temperature for both CONV-EP and EP-SCE were fixed at $5 \mathrm{~mA} / \mathrm{cm}^{2}$ and $40{ }^{\circ} \mathrm{C}$, respectively. At least three gold films were electroplated at each experimental conditions to confirm the repeatability.

\subsection{Micro-compression tests}

Micro-size compression pillars were fabricated from three pieces each of the Au films prepared by the CONV-EP and the EP-SCE with a film thickness of $\sim 50 \mu \mathrm{m}$. FIB (Hitachi FB2100) was utilized to fabricate the non-tapered $\mathrm{Au}$ micro-pillars. Dimensions of the micro-pillars fabricated were $10 \times 10 \times 20 \mu \mathrm{m}^{3}$. Micro-compression tests were conducted with a testing machine specially designed for micro-size specimens, which a diamond flat-punch indenter equipped to a load cell was used as the compression platen [16]. The micro-compression was controlled at a constant displacement rate of $0.1 \mu \mathrm{m} / \mathrm{s}$ using a piezo-electric actuator.

\subsection{Characterization}

Crystalline structures of the Au films were characterized using grazing incidence X-ray diffraction (GIXRD, Rigaku Ultima) at a glancing angle of $3^{\circ}$. Surface morphologies of the electroplated Au films were examined by a scanning electron microscope (SEM, JEOL 7500F) and an atomic force microscope (AFM, Park system XE-100). Observation of the microstructure and the deformation behavior of the Au micro-pillars was conducted using a scanning ion microscope (SIM) equipped with the FIB. Grain boundary characteristics of the pillar surface normal to the compression axis were examined by electron backscatter diffraction (EBSD, INCA Crystal software, Oxford Instruments) installed in a SEM (SU4300SE, Hitachi), and carbon content in the Au films was examined by energy-dispersive X-ray spectroscopy (EDS) equipped in the same SEM. 


\section{Results and discussion}

Fig. 1(a)-(d) show surface morphology and roughness of the CONV-EP and the EP-SCE films with a thickness of $\sim 5 \mu \mathrm{m}$. The CONV-EP film has bubble-clustered-bumps morphology, and the film roughness $\left(R_{\mathrm{a}}\right)$ was about $12-13 \mathrm{~nm}$. Surface morphology and $R_{a}$ of the EP-SCE film were very similar to that of the CONV-EP film. It is noticed that the $\mathrm{Au}$ electrolyte used in this study contained traces amount of a surface brightener, sodium gluconate, which can give the leveling effects [20]. $R_{\mathrm{a}}$ of the Cu plates was $19.8 \mathrm{~nm}$. Hence, high surface smoothness was obtained for the CONV-EP film, and it became difficult for the EP-SCE to further improve the surface smoothness.

Fig. 2 shows the GIXRD patterns of the CONV-EP and the EP-SCE Au films. Both films exhibit the characteristic peaks corresponding to the (111), (200), (220), and (311) planes of metallic face-centered cubic gold. The major diffraction peak is (111) for both Au films, which is because the surface energy of the (111) crystal growth orientation is the lowest when compared with other crystal orientations [21]. On the other hand, the diffraction peaks of the EP-SCE Au film are broader than those of the CONV-EP film. The grain refinement effect is suggested to be contributed by the same phenomena observed in electrodeposition of $\mathrm{Ni}$, which are the periodic-plating-characteristics (PPC) [11] and the co-deposited carbon contents [16]. The EDS result showed that the carbon content in the EP-SCE film was $39.92 \pm 2.02$ at.\%, which was higher than that in the CONV-EP film (29.63 \pm 3.11 at.\%). The co-deposited carbon would directly contribute to the grain refinement effect and immobilize movement of the grain boundaries [19], which is especially important in maintaining the ultra-fine grain structure since the mechanical strength is closely related to the grain size. The hydrobaric effect, which is the effect of pressure on crystal structure of the materials electrodeposited in an aqueous electrolyte, is also expected to have influences on the Au crystal structure [22].

Fig. 3 shows SIM images of the micro-pillars fabricated respectively from the 
CONV-EP and the EP-SCE Au thick films before and after the micro-compression tests. The

CONV-EP pillar showed camouflage-like patterns on the side-surface, where clear boundaries along the patterns with different level of brightness would be the grain boundaries. On the other hand, inconspicuous grain boundaries were observed on the surface of the EP-SCE Au pillars, which implied the grain size was finer than ca. $1 \mu \mathrm{m}$ [23]. After the micro-compression test, barrel-shape deformation was observed in the middle part of both CONV-EP and EP-SCE pillars. The deformation following the slips of grain boundaries or formation of barrel-shape is well recognized to be the typical characteristics of the deformation of poly- and nano-crystalline structures [24]. These results confirmed the polycrystalline structure in the two pillars. In addition to the rough observation of grain size from SIM, EBSD measurement was conducted to confirm the grain size distribution in the Au films. Fig. 4(a) shows the orientation map of the CONV-EP pillar determined from the EBSD analysis. The grains randomly distribute with a wide range of various orientations, and no obviously preferred-growth orientation was observed. The corresponding grain size distribution is summarized in Fig. 4(b), which the grain size ranged from few hundred nanometer to $1-2 \mu \mathrm{m}$ with an average of $\sim 0.8 \mu \mathrm{m}$. It should be noticed that grains size of the EP-SCE Au was too small to be determined by EBSD measurement; therefore, the average grain size of the EP-SCE Au was estimated from the GIXRD pattern (Fig. 2) using the Scherrer equation. The estimated average grain size of the EP-SCE Au was extremely small of $\sim 13 \mathrm{~nm}$, which was far smaller than that of the CONV-EP Au.

Mechanical strengths of the Au films were determined from engineering stress-strain (SS) curves generated from the micro-compression tests. Fig. 5 shows the SS curves of the CONV-EP and the EP-SCE micro-pillars. Yield strength of the CONV-EP pillar was estimated to be $380 \mathrm{MPa}$, which was determined by the cross-point of the SS curve and $0.2 \%$ offset line of the elastic deformation region. The yield strength obtained from the EP-SCE pillar was evaluated to be $520 \mathrm{MPa}$ and was much higher than that of the CONV-EP pillar. 
Moreover, the compressive flow stress reached $~ 800 \mathrm{MPa}$ for the EP-SCE pillar, further suggesting that mechanical properties of electroplated Au can be enhanced by employing the EP-SCE method. The significant enhancement of the mechanical properties in the EP-SCE Au pillar could be explained by the Hall-Petch relationship [5], that is, strength of polycrystalline metallic materials is enhanced following a decrease in the grain size. As confirmed by the GIXRD patterns (Fig. 2) and the SIM images (Fig. 3), the EP-SCE Au film had a finer grain size when compared with the CONV-EP film.

It is worth noting that the high mechanical strength is attributed to the ultra-fine grains. For the EP-SCE, the PPC, the hydrobaric effect, and the high carbon content could all contribute to the grain refinement effect. Besides, the high carbon content suggests that the EP-SCE films are promising electronic materials for the fabrication process involving heat treatment since the grain growth would be suppressed.

\section{Conclusions}

An enhancement in the mechanical properties of the Au films electroplated with the SCE was confirmed by micro-compression tests in this study. Also, grain refinement effect was observed in the Au films prepared by the EP-SCE. Also, a high carbon content was observed in the EP-SCE film, which was one of the causes of the grain refinement effect. The yield strength and compressive flow stress of the EP-SCE micro-pillar with dimensions of $10 \times 10 \times 20 \mu \mathrm{m}^{3}$ reached $520 \mathrm{MPa}$ and $\sim 800 \mathrm{MPa}$, respectively. The fine grains were suggested to be the main cause of the enhancement in the mechanical properties based on the grain-boundary hardening mechanism known as the Hall-Petch relationship. The present study demonstrated an alternative EP method in the fabrication of Au materials with improved mechanical properties for applications in electronic devices such as MEMS devices. 
189 Acknowledgement

190 This work is supported by CREST Project operated by the Japan Science and Technology

191 Agency (JST).

192 


\section{References}

[1] Y. Okinaka, M. Hoshino, Some recent topics in gold plating for electronics applications, Gold Bull. 31 (1998) 3-13.

[2] T.A. Green, Gold electrodeposition for microelectronic, optoelectronic and microsystem applications, Gold Bull. 40 (2007) 105-114.

[3] D. Yamane, T. Konishi, T. Matsushima, K. Machida, H. Toshiyoshi, K. Masu, Design of sub-1g microelectromechanical systems accelerometers, Appl. Phys. Lett. 104 (2014) 074102. [4] J.R. Greer, W.C. Oliver, W.D. Nix, Size dependence of mechanical properties of gold at the micron scale in the absence of strain gradients, Acta Mater. 53 (2005) 1821-1830.

[5] N.J. Petch, The cleavage strength of polycrystals, J. Iron Steel Inst., 174 (1953) 25-28.

[6] Y. Wang, M. Chen, F. Zhou, E. Ma, High tensile ductility in a nanostructured metal, Nature 419 (2002) 912-915.

[7] S. Cheng, E. Ma, Y. Wang, L. Kecskes, K. Youssef, C. Koch, U. Trociewitz, K. Han, Tensile properties of in situ consolidated nanocrystalline Cu, Acta Mater. 53 (2005) $1521-1533$.

[8] M. Stern, A.L. Geary, Electrochemical Polarization: I . A Theoretical Analysis of the Shape of Polarization Curves, J. Electrochem. Soc. 104 (1957) 56-63.

[9] M. S. Chandrasekar, M. Pushpavanam, Pulse and pulse reverse plating conceptual, advantages and applications, Electrochim. Acta 53 (2008) 3313-3322.

[10] C.-Y. Chen, M. Yoshiba, T. Nagoshi, T.-F.M. Chang, D. Yamane, K. Machida, K. Masu, M. Sone, Pulse electroplating of ultra-fine grained Au films with high compressive strength, Electrochem. Commun. 67 (2016) 51-54.

[11] T.-F.M. Chang, M. Sone, A. Shibata, C. Ishiyama, Y. Higo, Bright nickel film deposited by supercritical carbon dioxide emulsion using additive-free Watts bath, Electrochim. Acta 55 (2010) 6469-6475. 
[12] T.-F.M. Chang, T. Shimizu, C. Ishiyama, M. Sone, Effects of pressure on electroplating (2013) 25-28.

[13] T.-F.M. Chang, T. Tasaki, C. Ishiyama, M. Sone, Defect-free nickel micropillars fabricated at a high current density by application of a supercritical carbon dioxide emulsion, Ind. Eng. Chem. Res. 50 (2011) 8080-8085.

[14] J. Ke, W. Su, S.M. Howdle, M.W. George, D. Cook, M. Perdjon-Abel, P.N. Bartlett, W.

Zhang, F. Cheng, W. Levason, G. Reid, J. Hyde, J. Wilson, D.C. Smith, K. Mallik, P. Sazio,

Electrodeposition of metals from supercritical fluids, PNAS 106 (2009) 14768-14772.

[15] J.M. Blackburn, D.P. Long, A. Cabanas, J.J. Watkins, Deposition of conformal copper and nickel films from supercritical carbon dioxide, Science 294 (2001) 141-145.

[16] T. Nagoshi, T.-F.M. Chang, S. Tatsuo, M. Sone, Mechanical properties of nickel fabricated by electroplating with supercritical $\mathrm{CO}_{2}$ emulsion evaluated by micro-compression test using non-tapered micro-sized pillar, Microelectron. Eng. 110 (2013) 270-273.

[17] S.-T. Chung, W.-T. Tsai, Nanocrystalline Ni-C electrodeposits prepared in electrolytes containing supercritical carbon dioxide, J. Electrochem. Soc. 156 (2009) D457-D461. Mater. 25 (1991) 955-958.

[19] T. Nagoshi, T.-F. M. Chang, T. Sato, M. Sone, Effect of annealing on mechanical properties of nickel electrodeposited using supercritical $\mathrm{CO}_{2}$ emulsion evaluated by micro-compression test, Microelectron. Eng. 153 (2016) 101-104.

[20] L. Oniciu, L. Mureşan, Some fundamental aspects of levelling and brightening in metal electrodeposition, J. Appl. Electrochem. 21 (1991) 565-574.

[21] D. Udler, D.N. Seidman, Grain boundary and surface energies of fcc metals, Phys. Rev. B 54, 54 (1996) R11133-R11136. 
245 [22] W.-H. Lin, C.-Y. Chen, T.-F.M. Chang, Y.-J. Hsu, M. Sone, Effects of Pressure in 246 Cathodic Deposition of $\mathrm{TiO}_{2}$ and $\mathrm{SnO}_{2}$ with Supercritical $\mathrm{CO}_{2}$ Emulsified Electrolyte, 247 Electrochim. Acta 208 (2016) 244-250.

248 [23] J.M. Chabala, R. Levi-Setti, Y.L. Wang, Practical resolution limits of imaging 249 microanalysis with a scanning ion microprobe, Appl. Surf. Sci. 32 (1988) 10-32.

250 [24] M. Dietiker, S. Buzzi, G. Pigozzi, J.F. Löffler, R. Spolenak, Deformation behavior of 251 gold nano-pillars prepared by nanoimprinting and focused ion-beam milling, Acta Mater. 59 252 (2011) 2180-2192. 
254 Figure 1. SEM images of (a) the CONV-EP (CD $\left.=5 \mathrm{~mA} / \mathrm{cm}^{2}\right)$ Au film and (b) the EP-SCE $255\left(\mathrm{CD}=5 \mathrm{~mA} / \mathrm{cm}^{2}, P_{\mathrm{scCO} 2}=10 \mathrm{MPa}\right)$ Au film. AFM micrographs of Au film surface prepared 256 by (c) the CONV-EP and (d) the EP-SCE.

257

Figure 2. GIXRD patterns of the CONV-EP and the EP-SCE Au films.

259

Figure 3. SIM images of the CONV-EP Au pillar (a) before and (b) after the compression test and the EP-SCE pillar (c) before and (d) after the micro-compression test.

Figure 4. (a) EBSD orientation map of the CONV-EP Au pillar with the view of compression direction. (b) Grain size distribution obtained from (a).

265

266

Figure 5. Engineering SS curves of the Au micro-pillars fabricated from the CONV-EP and 267 the EP-SCE Au films. The dimensions of the two pillars are $10 \times 10 \times 20 \mu \mathrm{m}^{3}$. 

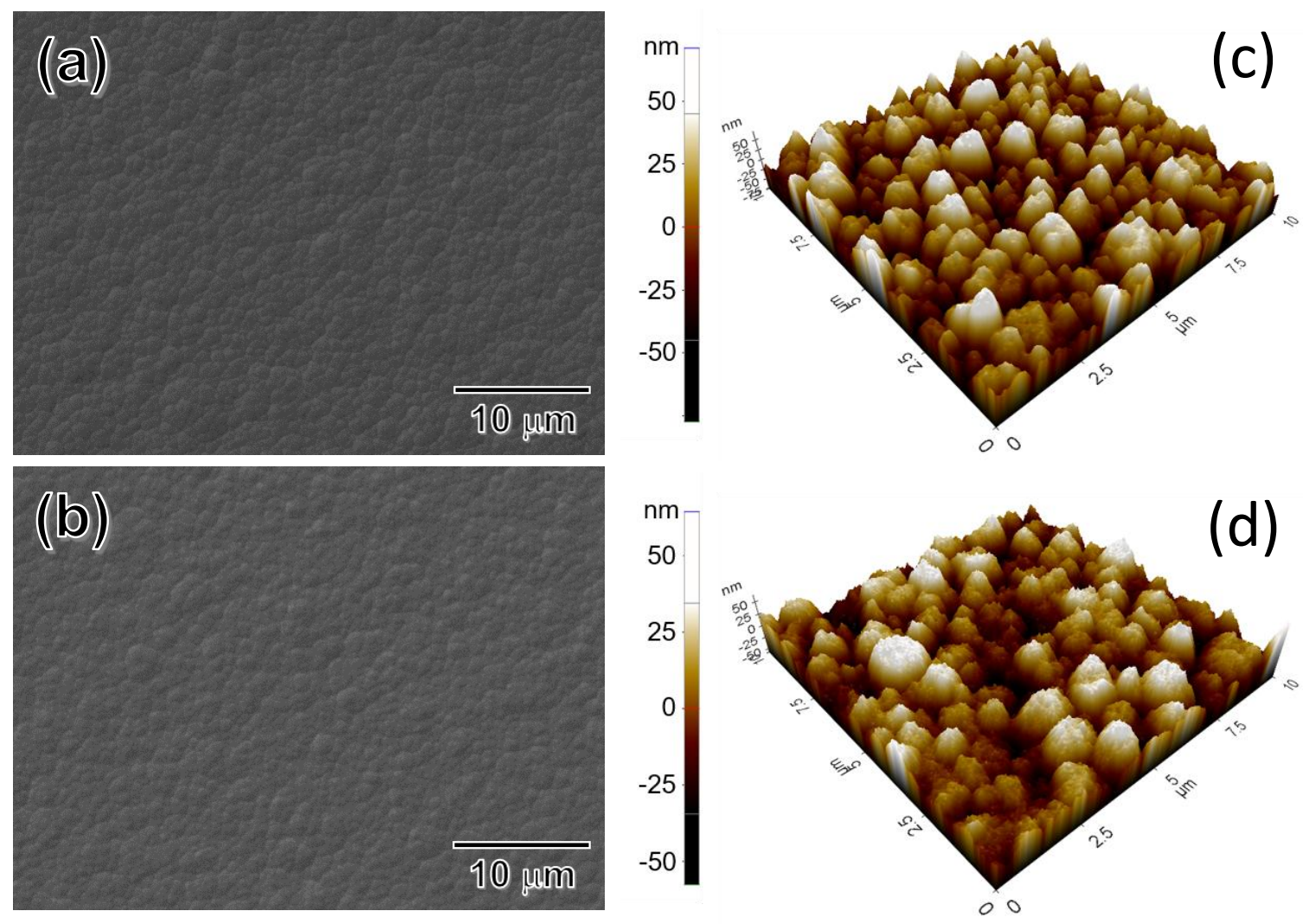

Figure 1 SEM images of (a) the CONV-EP $\left(C D=5 \mathrm{~mA} / \mathrm{cm}^{2}\right)$ $\mathrm{Au}$ film and (b) the EP-SCE $\left(\mathrm{CD}=5 \mathrm{~mA} / \mathrm{cm}^{2}, P_{\mathrm{scCO} 2}=10\right.$ $\mathrm{MPa}) \mathrm{Au}$ film. AFM micrographs of Au film surface prepared by (c) the CONV-EP and (d) the EP-SCE. 


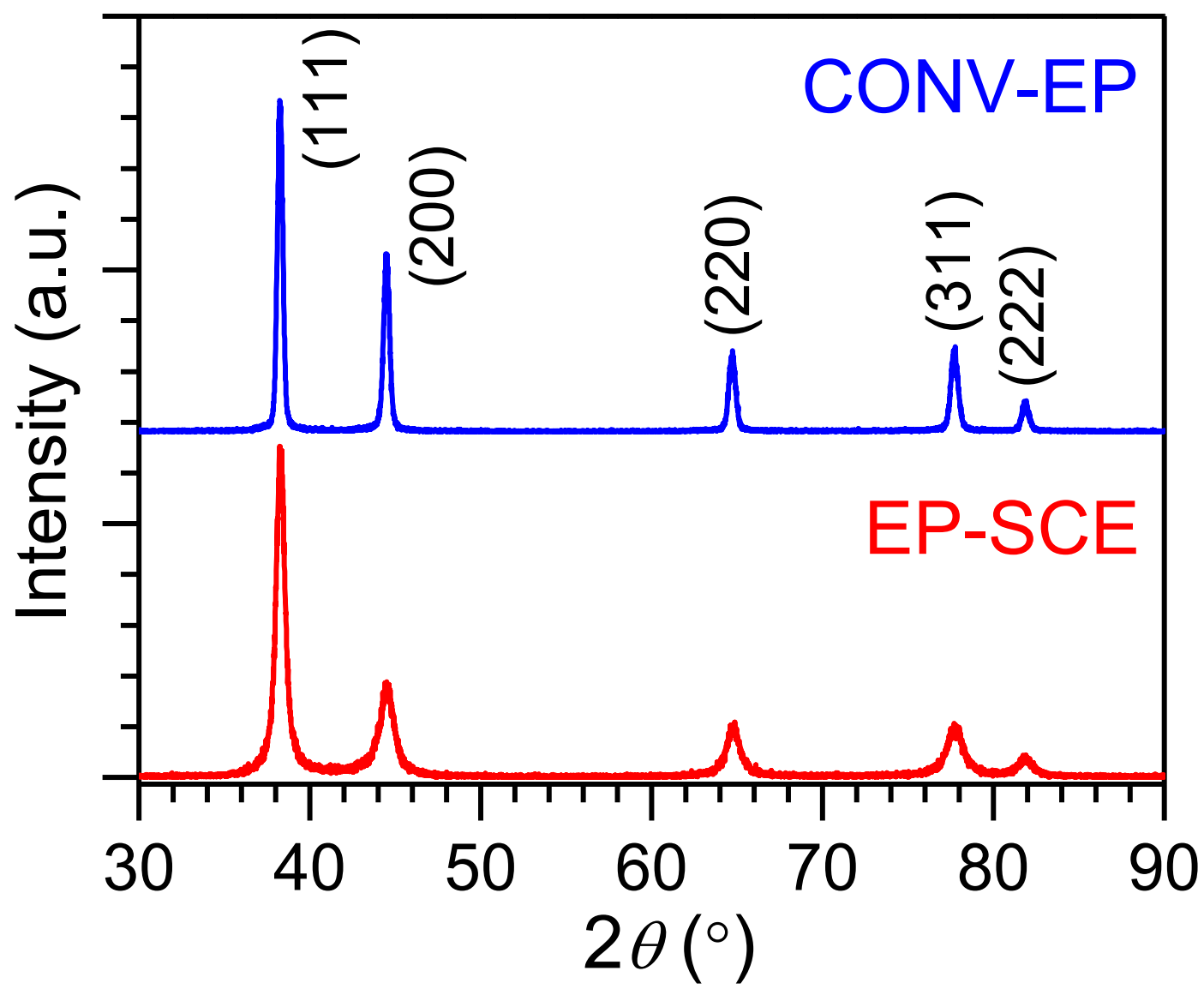

Figure 2 GIXRD patterns of the CONV-EP and the EP-SCE Au films. 


\section{(a)}

\section{(b)}

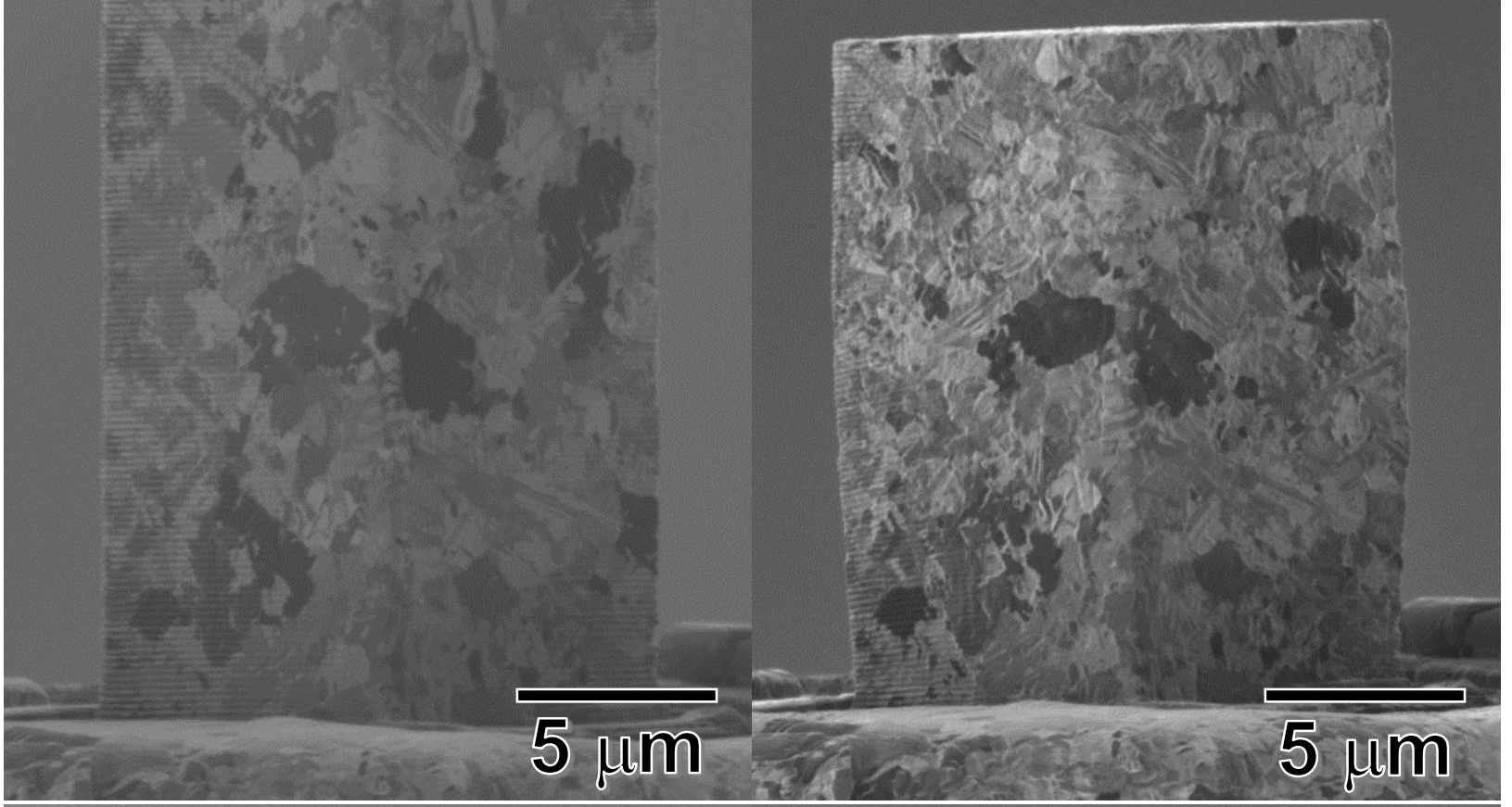

(c)

(d)

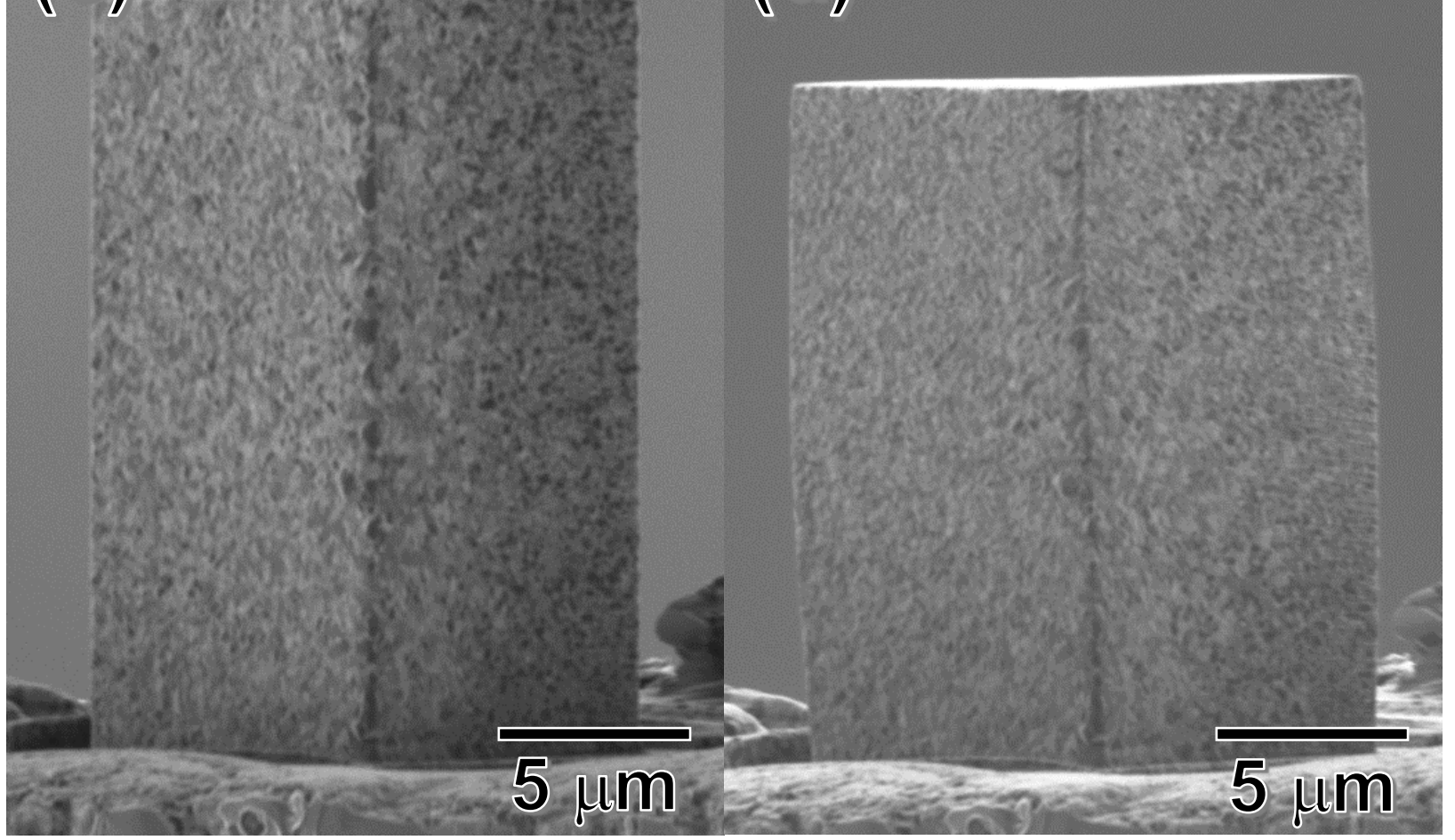

Figure 3 SIM images of the CONV-EP Au pillar (a) before and (b) after the compression test and the EP-SCE pillar (c) before and (d) after the micro-compression test. 


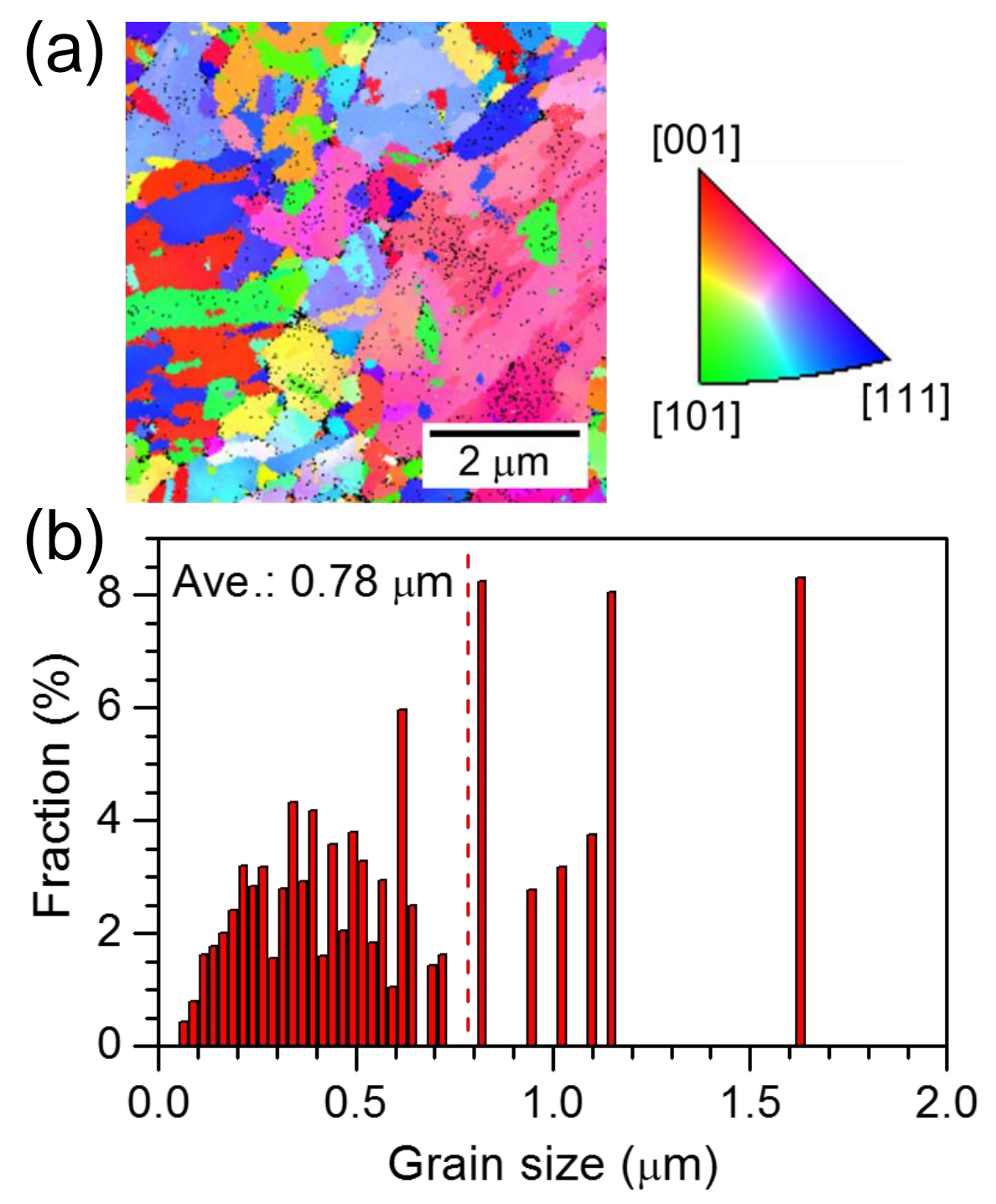

Figure 4 (a) EBSD orientation map of the CONV-EP Au pillar with the view of compression direction. (b) Grain size distribution obtained from (a). 


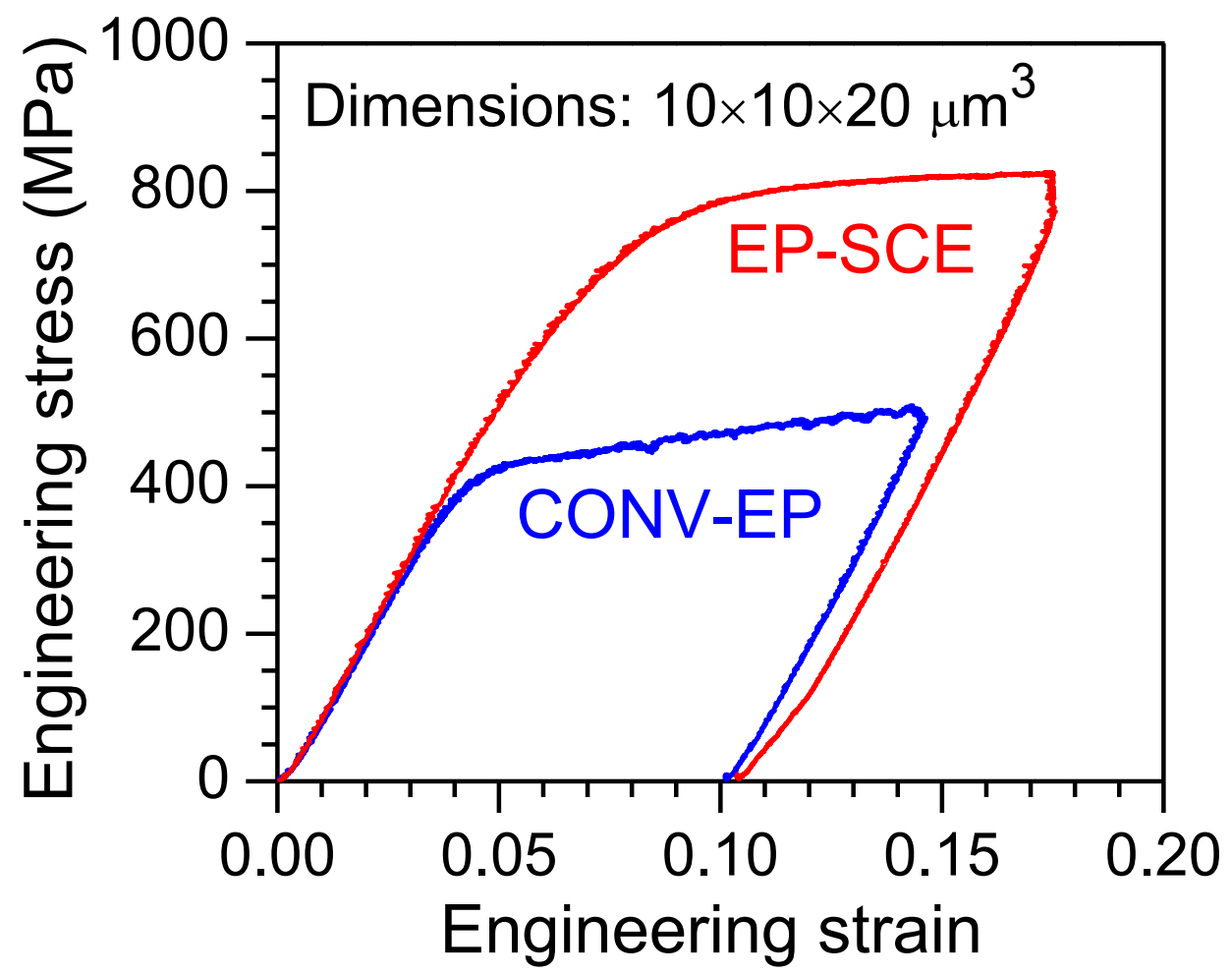

Figure 5 Engineering $S S$ curves of the Au micro-pillars fabricated from the CONV-EP and the EP-SCE Au films. The dimensions of the two pillars are $10 \times 10 \times 20 \mu \mathrm{m}^{3}$. 


\section{Graphical abstract}

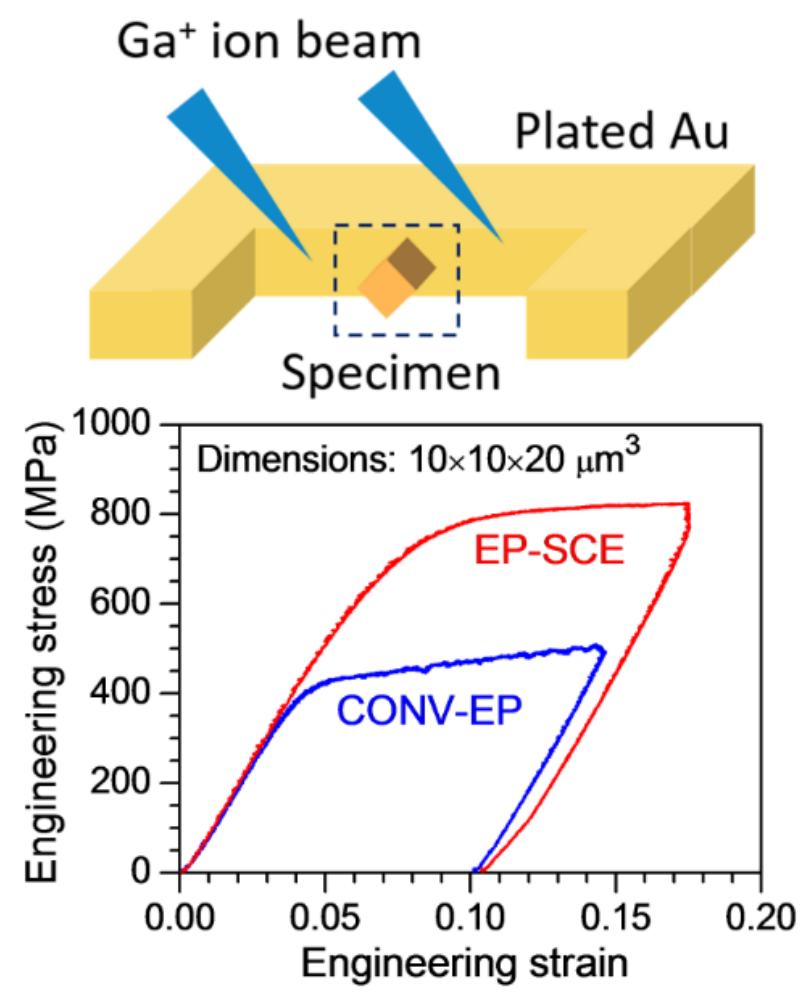

As-fabricated Compressed

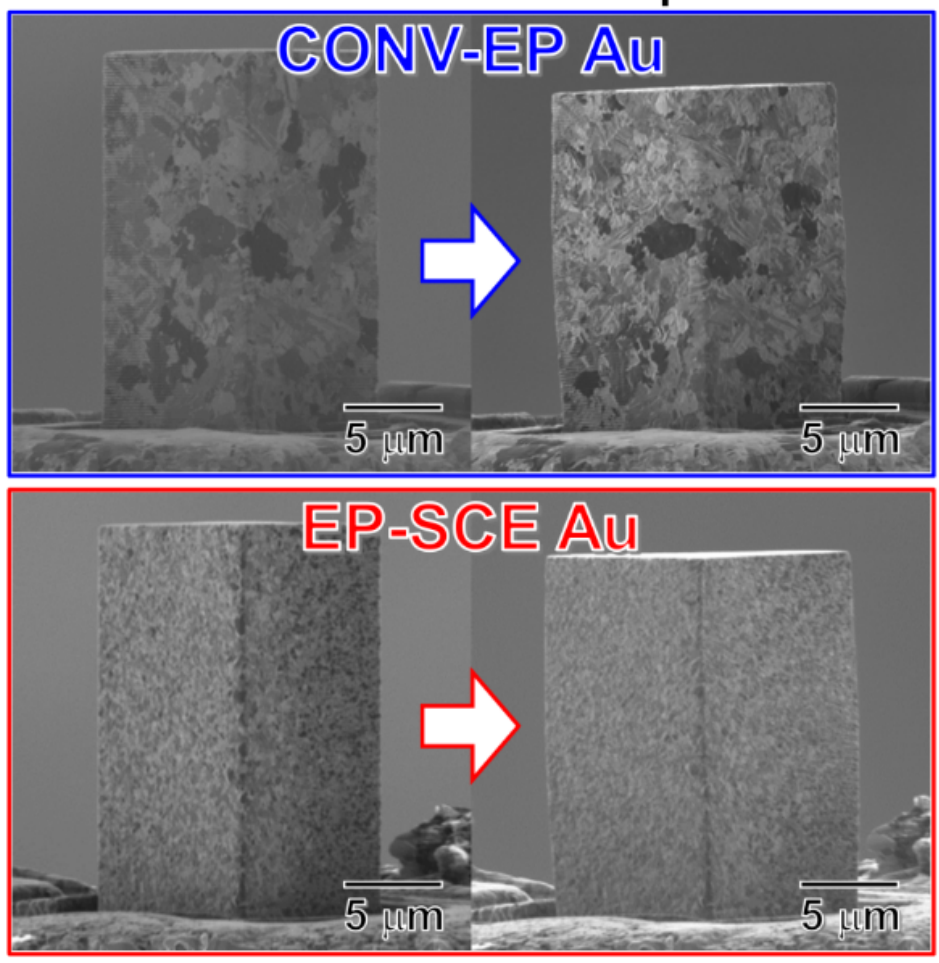

